\title{
DISORDERS OF COAGULATION STATUS AND HAEMOSTASIS AS PROGNOSTIC PARAMETERS OF IMMEDIATE AND EARLY RESULTS AFTER SURGICAL MYOCARDIAL REVASCULARISATION
}

\author{
Dragan Milić1 ${ }^{1,2}$, Milan Lazarević ${ }^{1}$, Dragan Bogdanovićc ${ }^{3}$ Zoran Damnjanović ${ }^{4}$, Saša Živić ${ }^{1}$, \\ Dejan Perić ${ }^{1}$, Aleksandar Kamenov ${ }^{1}$, Vladimir Stojiljković ${ }^{1}$, Mladjan Golubović
}

\begin{abstract}
Surgical myocardial revascularization is one of the most commonly performed surgical procedures in the world. Over time, with the development of technology and modern diagnostic procedures, as well as the advancement of surgical techniques, the mortality rate for elective uncomplicated cases has fallen to below $2 \%$. Nevertheless, despite the exceptional development of the surgical techiniques, the rate of postoperative complications, that can compromise the patients, is over $10 \%$. The aim of this study was to define a group of patients with an increased risk of postoperative complications depending on the disorders of coagulation status and haemostasis.

Twenty eight patients who underwent surgical revascularization of the myocardium were included in this prospective, non-randomized study. The study was conducted at the Clinic for Cardiac Surgery, Clinical Center Nis, from January to April 2017. Preoperatively as well as 3 hours, 24 hours, 48 hours, 3 days, and 5 days postoperatively, the following parameters were determined: blood count, inflammation parameters ( $C$ reactive protein, presepsin); coagulation status (prothrombin time (PT), International Normalized Ratio (INR), activated partial thromboplastin time (APTT), fibrinogen, anti-thrombin III, D dimer).

The only preoperative independent prognostic parameter for increased postoperative drainage was INR. Activated clothing time (ACT) was an independent postoperative prognostic parameter of increased postoperative drainage, probably due to delayed or prolonged heparin activity. Inflammation parameters showed no association with the onset of postoperative complications. In relation to patients without bleeding, in patients with bleeding, significantly higher values of urea and the difference in APTT values, preoperatively and at the end of the monitoring period, were detected. Multivariate logistic regression analysis, confirmed the difference in APTT values preoperatively and at the end of the monitoring period, as the only factor, significantly associated with the risk of bleeding. Multivariate linear regression analysis, confirmed the value of the urea, as the only factor significantly associated with the change in total allogeneic transfusion value. Increase in urea levels is associated with an increase in the total amount of allogeneic transfusion. Correlation analysis showed that the increased number of days in the intensive care was significantly associated with female gender, number of grafts, prolonged ECC, clamping time, hematocrit (HCT), PT, INR preoperatively and at the end of the follow-up period.

Surgical myocardial revascularization is a safe method with a minimal morbidity rate. Using of modern methods for the preoperative monitoring of haemostasis may significantly reduce the risk of postoperative bleeding and the need for transfusion of red blood cells and other blood derivatives.
\end{abstract}

Acta Medica Medianae 2019;58(1):64-81. factors

Key words: cardiosurgery, coagulation status, inflammation parameters, risk

\footnotetext{
${ }^{1}$ Department of Cardiac Surgery, Clinical Centre of Niš, Niš, Serbia

${ }^{2}$ University of Nis, Faculty of Medicine, Niš, Serbia

Institute for Public Health Niš, Niš, Serbia

${ }^{4}$ Department of Vascular surgery, Clinical Centre of Niš, Niš, Serbia

${ }^{5}$ Department of Anesthesiology, Clinical Centre of Niš, Niš, Serbia
}

\section{Introduction}

Cardiovascular (CV) diseases are leading cause of death in most developed countries and in many undeveloped countries. Epidemiological data indicate that CV diseases cause almost half of total mortality, while malignant tumors and lung diseases are represented by $22 \%$ and $10 \%$, respectively. Every year, over 17 million people die from cardiovascular dise- 
ases in the world, which is a third of the population dying $(1,2)$.

Cardiovascular diseases include: ischemic heart disease (stable angina pectoris, acute coronary syndrome, sudden cardiac death, cardiac insufficiency), acute stroke (ischemic stroke and haemorrhagic stroke), and peripheral arterial occlusive disease.

According to World Health Organization (WHO) estimation, the dying structure from cardiovascular diseases is as follows: ischemic heart disease is on average $41 \%$, stroke with $32 \%$ and other heart disease with $27 \%$ (3). In our country the situation is significantly different: slightly more than half of those who died of CV disease had a diagnosis of one of the heart diseases, while the participation of the stroke was $29 \%$ (4).

Coronary artery disease (coronary or ischemic heart disease) is the most common cause of death, leading to death, disability and economic losses more than any other illness, despite a significant reduction in mortality over the past three decades. The frequency of may be been seen from the results of large studies, according to which, the risk of the onset of coronary artery disease during life, for a man at the age of 40 , is almost $40 \%$ (3).

The role of the surgeon in the process of CV disease treatment, finds its significant place from the moment when it came to the knowledge that obstructive atherosclerotic lesions on the coronary arteries are directly responsible for the onset of coronary disease. Aortocoronary bypass is a procedure that is carried out at the heart in order to overcome narrowing of blood vessels that nourish the heart. The results of the conducted research show that in the United States alone, 1500000 patients suffer from coronary heart disease annually, while surgical myocardial revascularization is performed in almost every fourth patient. A similar trend was observed in the countries of Western Europe (1,000 revascularization per million inhabitants), while in our country this number is significantly lower (600 revascularization per million inhabitants) (5).

Although surgical revascularization of the myocardium is now the most commonly performed surgical procedure in general, with mortality of about $1 \%$ in elective cases, there is still a high risk of emergency surgery in patients with acute coronary syndrome (6). Previous studies suggest that surgical revascularization of the myocardium has been a method of choice for the treatment of most patients with coronary heart disease for more than 50 years. The results of surgical treatment are superior in comparison with empirical medication therapy, in almost all investigated subgroups of patients. After performing this surgical procedure, a longer survival, longer period without new myocardial infarction, and significant improvement in the quality of life is achieved, while the incidence of the new chest pain onset is lower and the working capacity of the majority of operatives is excellent. It is therefore important to note that there are many benefits of early surgical revascularization, including limiting of infarction expansion, avoiding left ventricular dysfunction and cardiac insufficiency. The underlying risk lies in the ischemic-reperfusion myocardial damage, which can lead to the appearance of a hemorrhagic infarction, with all of its complications $(5,7,8)$.

\section{Material and methods}

The aim of this study was to examine the correlation between mortality, bleeding and the need for transfusion, the length of hospitalization and the presence of postoperative complications (respiratory, renal and hepatic) in relation to parameters of coagulation and fibrinolysis activation, platelet function disorders and inflammatory parameters, in patients who underwent surgical myocardial revascularization.

According to the statistical tests and calculations in the G-Power software package, this study included 28 patients who underwent surgical myocardial revascularization at the Clinic for Cardiovascular with Transplantation Surgery KC Niš, in the period from January to April 2017, which is reduced the probability of a error to a level of significance $r<$ 0.05 with a defined power of study of $80 \%$.

Preoperatively as well as 3 hours, 24 hours, 48 hours, 3 days, and 5 days postoperatively, the following parameters were determined:

1. blood count (erythrocyte count, hemoglobin, hematocrit, leukocyte count, platelet count);

2. Inflammation parameters ( $C$ reactive protein, presepsin);

3. coagulation status (prothrombin time, International Normalized Ratio, activated partial thromboplastin time, fibrinogen, anti-thrombin III, D dimer);

4. parameters of thrombocyte function (platelet activation adenosine di-phosphate (ADP HS), platelet activation with arachidonic acid (ASPI));

5. Rotational thrombelastometry parameters (direct activation of the thrombin peptide receptor (TRAP), internal and external coagulation pathway (clotting time, maximal clotting firmness, clot amplitude after 10 minutes, alpha angle, maximum lysis, functional fibrinogen).

The correlation of the above parameters with comparison to the duration of the extracorporeal circulation and the duration of the aortic cross clamp is determined.

\section{Statistical analysis}

Quantitative statistical analysis was carried out on a computer. Excel program from the Microsoft Office 2010 software package was used for typing, ranking, grouping, table and graphical data presentation. The calculations were made using the SPSS program in version 18.0 .

The following statistical parameters were displayed: arithmetic mean (AS), standard deviation (SD) and structure index (\%).

Comparison of mean values of numerical features between groups of patients with and without bleeding was performed by Student's T test or ManWhitney $U$ test, in cases where the distribution of values did not meet the requirements of normal 
schedule. Comparison of mean values of numerical features in the same patients between two measurements was done by the Paired-samples t test.

A comparison of the frequency of attributes between groups was performed by Mantel-Hensel's Quadratic Test (Mantel-Haenszel Chi square test) or Fisher exact test of the exact probability of a zero hypothesis (Fisher exact test) when one of the expected frequency of the mark was less than five. Changes in the value of the mark during the followup period were examined by Repeated measures ANOVA.

The study of the relationship between the amount of allogeneic transfusion, the number of days of total hospitalization, the number of days of hospitalization in intensive care, total drainage and all other investigated features was carried out by Spearman's rank correlation.

Determination of significant bleeding predictors was performed by multivariate logistic regression analysis. The odds ratio (OR) and the 95\% confidence interval (CI) were calculated. By using the Wald method step-by-step (Backward: Wald) from a mul- tivariate model, all factors, the statistical significance of which, was not confirmed were excluded.

Multivariate linear regression analysis was used to determine factors significantly related to the values of allogeneic transfusion, the number of days of hospitalization overall, the number of days in intensive care and total drainage. The values of regression coefficients (B) and the limits of their $95 \%$ confidence interval were calculated. Using stepwise methods in final models, only those factors that are significantly related to dependent variables are retained. As a threshold of statistical significance in the conclusion, the estimation error was less than 5\% ( $p$ $<0.05$ ). The statistical analysis results were showed as tables and graphically.

\section{Results}

The study included 28 patients, 22 men (79\%) and 6 women $(21 \%)$, the average age of 64 years. The basic characteristics of the respondents are shown in Table 1.

Table 1. The basic characteristics of the respondents

\begin{tabular}{|lc||}
\hline \multicolumn{1}{|c|}{ Characteristic } & Value \\
\hline \hline Age & $64.14 \pm 6.85$ \\
\hline Gender & \\
\hline Male & $22(78.6)$ \\
Female & $6(21.4)$ \\
Smoking & $6(21.4)$ \\
Diabetes & $13(46.4)$ \\
\hline Regulation of diabetes & \\
\hline Without regulation & $1(3.6)$ \\
Oral & $6(21.4)$ \\
Insulin & $6(21.4)$ \\
BMI & $29.94 \pm 4.4$ \\
Triglycerides & $1.86 \pm 0.85$ \\
Cholesterol & $4.36 \pm 2.12$ \\
Urea & $6.38 \pm 1.46$ \\
Kreatinin & $95.67 \pm 18.34$ \\
EF percentage & $53.86 \pm 10.7$ \\
Surgery type (number of grafts) & $2.57 \pm 0.79$ \\
Duration extra corporal ECC & $102.36 \pm 22.22$ \\
Time of clamping & $45.79 \pm 11.63$ \\
The amount of given & $1158.93 \pm 278.57$ \\
\hline Surgery type (number of grafts) & \\
\hline CABG I & $1(3.6)$ \\
CABG II & $13(46.4)$ \\
CABG III & $12(42.9)$ \\
CABG IV & $1(3.6)$ \\
CABG V & $1(3.6)$ \\
Defibrilation & $26(92.9)$ \\
Diuresis & $1346.43 \pm 569.26$ \\
ACT at admittance & $138.96 \pm 13.33$ \\
ACT afterAH & $584.46 \pm 104.5$ \\
ACT after AP & $125.79 \pm 12.19$ \\
Autotransfusion & $569.11 \pm 124.27$ \\
Hospitalization in intensive care unit & $4.54 \pm 1.29$ \\
Hospitalization in semi-intensive care & $2.43 \pm 1.4$ \\
unit & $6.96 \pm 0.51$ \\
Total hospitalization & $583.33 \pm 224.11$ \\
Allogenic transfusion total & \\
Drainage total & \\
Diuresis total & \\
\hline NOTE: values are displayed as arithmetic mean & \\
\hline
\end{tabular}


Table 2. Application of allogeneic transfusion, blood derivatives, voluven and crystalloids, drainage and diuresis per follow-up days

\begin{tabular}{|c|c|c|c|c|c|c|c|c|}
\hline & I day & II day & III day & IV day & V day & VI day & VII day & Total \\
\hline $\begin{array}{l}\text { Allogenic } \\
\text { Transfusion }\end{array}$ & $\begin{array}{c}3 \\
(933 \pm \\
728.6) \\
\end{array}$ & $\begin{array}{c}\mathbf{2} \\
(350.0 \pm \\
0.0) \\
\end{array}$ & $\begin{array}{c}\mathbf{1} \\
(700.0 \pm \\
0.0) \\
\end{array}$ & $\begin{array}{c}\mathbf{4} \\
(350.0 \pm \\
0.0) \\
\end{array}$ & - & $\begin{array}{c}\mathbf{2} \\
(350.0 \pm \\
0.0)\end{array}$ & $\begin{array}{c}\mathbf{2} \\
(350.0 \pm \\
0.0) \\
\end{array}$ & $\begin{array}{c}\mathbf{2} \\
(583 \pm 224)\end{array}$ \\
\hline $\begin{array}{c}\text { Application of } \\
\text { plasma }\end{array}$ & - & - & - & - & - & - & - & - \\
\hline $\begin{array}{c}\text { Application } \\
\text { of cryo }\end{array}$ & - & - & - & - & - & - & - & - \\
\hline $\begin{array}{l}\text { Application of } \\
\text { thrombocytes }\end{array}$ & $\begin{array}{c}\mathbf{4} \\
(6.25 \pm \\
2.87) \\
\end{array}$ & - & - & - & - & - & - & $\begin{array}{c}\mathbf{4} \\
(6.25 \pm 2.87)\end{array}$ \\
\hline Crystaloids & - & - & - & - & - & - & - & - \\
\hline Drainage & $\begin{array}{c}\mathbf{2 8} \\
(414.3 \pm \\
207) \\
\end{array}$ & $\begin{array}{c}\mathbf{2 8} \\
(416.9 \pm \\
141.9) \\
\end{array}$ & $\begin{array}{c}\mathbf{1 8} \\
(190.8 \pm \\
140.6) \\
\end{array}$ & $\begin{array}{c}\mathbf{3} \\
(203.3 \pm \\
131.3) \\
\end{array}$ & - & - & - & $\begin{array}{c}\mathbf{2 8} \\
(976 \pm 388)\end{array}$ \\
\hline Diuresis & $\begin{array}{c}\mathbf{2 8} \\
(2779 \pm \\
764)\end{array}$ & $\begin{array}{c}\mathbf{2 8} \\
(3004 \pm \\
1406)\end{array}$ & $\begin{array}{c}\mathbf{2 8} \\
(2795 \pm \\
1263)\end{array}$ & $\begin{array}{c}\mathbf{1 7} \\
(2852 \pm \\
1205)\end{array}$ & $\begin{array}{c}9 \\
(3105 \pm \\
686)\end{array}$ & $\begin{array}{c}\mathbf{2} \\
(2850 \pm \\
0.0)\end{array}$ & $\begin{array}{c}\mathbf{1} \\
(1800 \pm \\
0.0)\end{array}$ & $\begin{array}{c}\mathbf{2 8} \\
(11577 \pm 3639)\end{array}$ \\
\hline
\end{tabular}

NOTE: values are displayed as number of patients (arithmetic mean \pm SD)

Allogeneic transfusion was administered in 3 patients on the first postoperative day, and the average blood amount was $933.3 \mathrm{ml}$ (Table 2). On the second day it was applied in two patients, $350 \mathrm{ml}$ each, the third day in one patient, $700 \mathrm{ml}$, the fourth day in 4 patients, $350 \mathrm{ml}$ each.

On the fifth postoperative day allogeneic transfusion was not applied to any patient. On the sixth and seventh day, allogeneic transfusion was administered in 2 patients, in $350 \mathrm{ml}$ each. In total, allogeneic transfusion was received by 12 patients, and the approximate amount of blood was $583 \mathrm{ml}$.

The drainage was measured for the first two days in all 28 patients, and the average value was $414.3 \mathrm{ml}$ during the first day and $416.9 \mathrm{ml}$ during the second day. On the third day, the drainage was measured in 18 patients, on average $190.8 \mathrm{ml}$, and on the fourth day in 3 patients on average $203.3 \mathrm{ml}$. After the fourth day, the drainage was not measured in any patient.

The diuresis was measured for the first three days in all 28 patients, and the average value was $2779 \mathrm{ml}, 3004 \mathrm{ml}$ and $2795 \mathrm{ml}$, by day. On the fourth day, the diuresis was measured in 17 patients, on average $2852 \mathrm{ml}$, on the fifth day in 9 patients on average $3105 \mathrm{ml}$, the six in 2 patients at $2850 \mathrm{ml}$, and on the seventh day in one patient, $1800 \mathrm{ml}$.

Platelets were administered only on the first day, in 4 patients with an average of 6.25 units, plasma, cryoprecipitate, volume and crystalloids were not applied.

The analysis of variance for repeated measurements showed that changes in the values of most of the features during monitoring were statistically significant (Table 3).

In almost all features, difference between preoperative values, values 2 hours after surgery and at the end of the follow-up period, was significant. In the period between 2 hours after the operation and at the end of the follow up period, the values of WBC, RBC, HGB and ACT decreased significantly, while the HCT and PLT values increased significantly. Compared to preoperative values, the values of MPV and INTEM CT were significantly lower than the preoperative values, until the values of CRP, P SEP, INR, fibrinogen, D dimer, ADP HS, ASPI, TRAP, EXTEM CT, EXTEM MCF , EXTEM A10, EXTEM ALPHA ANGLE, INTEM MCF, INTEM A10, INTEM ALPHA ANGLE, FIBTEM CT, FIBTEM MCF, FIBTEM A10, FIBTEM ALPHA ANGLE and troponin, were singnificantly higher.

Comparison of values in patients with and without bleeding, within the examined groups is shown in Table 4.

Diabetes was significantly more commonly reported in patients without bleeding (Table 4). In patients without bleeding., significantly higher BMI, triglycerides, cholesterol, ACT before surgery, PLT count preoperatively and INTEM ALPHA ANGLE were seen preoperativly, compare to patients with bleeding. In patients with bleeding, significantly higher values of the urea and difference in APTT preoperatively and at the end of the follow-up period, were detected compared to patients without bleeding. The values of all other features did not differ significantly in patients with and without bleeding. 
Table 3. Values of the blood count, inflammation parameters, coagulation status, platlets, rotational thrombelastometry, internal and external coagulation pathway

\begin{tabular}{|c|c|c|c|c|c|c|c|c|}
\hline Parameter & Preoperatively & $\begin{array}{c}2 \text { hours after } \\
\text { Surgery }\end{array}$ & $\begin{array}{c}24 \text { hours after } \\
\text { surgery }\end{array}$ & $\begin{array}{c}48 \text { hours after } \\
\text { surgery }\end{array}$ & $\begin{array}{c}72 \text { hours after } \\
\text { surgery }\end{array}$ & $\begin{array}{l}5 \text { days after } \\
\text { surgery }\end{array}$ & $\begin{array}{l}7 \text { days after } \\
\text { surgery }\end{array}$ & $\begin{array}{l}\text { Effect } \\
\text { time }\end{array}$ \\
\hline WBC & $7.2 \pm 1.6$ & $9.6 \pm 4.1$ & $9.2 \pm 3.0$ & $8.8 \pm 2.6$ & $7.6 \pm 1.9$ & $6.5 \pm 2.9$ & $7.1 \pm 2.7$ & $<<0.001$ \\
\hline RBC & $4.6 \pm 0.4$ & $4.2 \pm 0.5$ & $4.1 \pm 0.5$ & $3.6 \pm 0.4$ & $3.6 \pm 0.4$ & $3.7 \pm 0.4$ & $3.8 \pm 0.6$ & $<0.001$ \\
\hline HGB & $140.3 \pm 10.1$ & $121.0 \pm 10.7$ & $115.7 \pm 10.0$ & $103.5 \pm 10.5$ & $103.9 \pm 10.6$ & $105.1 \pm 11.0$ & $103.5 \pm 22.4$ & $<0.001$ \\
\hline HCT & $39.4 \pm 8.2$ & $34.7 \pm 6.9$ & $34.1 \pm 3.6$ & $30.6 \pm 3.5$ & $30.9 \pm 3.1$ & $41.0 \pm 50.5$ & $35.3 \pm 14.0$ & 0.272 \\
\hline PLT & $241.6 \pm 70.2$ & $175.0 \pm 50.0$ & $191.9 \pm 42.1$ & $160.7 \pm 50.5$ & $172.2 \pm 45.6$ & $236.6 \pm 59.1$ & $271.1 \pm 97.1$ & $<0.001$ \\
\hline MPV & $8.7 \pm 0.8$ & $7.8 \pm 0.7$ & $8.2 \pm 0.6$ & $11.2 \pm 15.1$ & $8.3 \pm 0.7$ & $7.7 \pm 0.7$ & $7.7 \pm 0.7$ & 0.209 \\
\hline CRP & $3.7 \pm 3.9$ & $4.6 \pm 4.2$ & $79.6 \pm 39.8$ & $188.9 \pm 61.9$ & $192.9 \pm 79.3$ & $125.6 \pm 73.4$ & $106.1 \pm 134.2$ & $<0.001$ \\
\hline P SEP & $177.6 \pm 59$ & $413.4 \pm 196.2$ & $461.6 \pm 207.6$ & $443.9 \pm 254.7$ & $496.1 \pm 353.3$ & $469.4 \pm 284.2$ & $504.1 \pm 296.9$ & $<0.001$ \\
\hline PT & $11.4 \pm 1.3$ & $18.8 \pm 30.8$ & $12.6 \pm 1.6$ & $12.2 \pm 1.4$ & $11.3 \pm 1.5$ & $16.3 \pm 23.3$ & $12.8 \pm 4.1$ & 0.444 \\
\hline INR & $1.1 \pm 0.1$ & $1.8 \pm 2.4$ & $1.2 \pm 0.2$ & $1.2 \pm 0.2$ & $1.1 \pm 0.1$ & $1.2 \pm 0.1$ & $1.2 \pm 0.4$ & 0.228 \\
\hline APTT & $26.4 \pm 3.3$ & $30.1 \pm 3.3$ & $38.1 \pm 52.0$ & $30.4 \pm 3.2$ & $30.9 \pm 5.9$ & $27.2 \pm 3.9$ & $27.5 \pm 4.0$ & 0.364 \\
\hline Fibrinogen & $4.1 \pm 2.0$ & $3.7 \pm 1.5$ & $5.1 \pm 0.9$ & $7.7 \pm 1.8$ & $18.9 \pm 24.0$ & $11.2 \pm 10.8$ & $9.2 \pm 2.2$ & 0.005 \\
\hline AT III & $100.6 \pm 14.1$ & $77.4 \pm 14.2$ & $75.0 \pm 13.6$ & $75.5 \pm 7.4$ & $77.5 \pm 11.6$ & $89.3 \pm 15.7$ & $96.2 \pm 17.0$ & $<0.001$ \\
\hline D dimer & $282 \pm 166.9$ & $431.2 \pm 852.6$ & $279.9 \pm 241.6$ & $426.5 \pm 856.7$ & $447.3 \pm 676.3$ & $788.8 \pm 982.3$ & $1450.9 \pm 1376.9$ & $<0.001$ \\
\hline ADP HS & $435.4 \pm 179.5$ & $424.0 \pm 199.0$ & $629.3 \pm 148.4$ & $582.7 \pm 125.2$ & $605.1 \pm 168.6$ & $645.4 \pm 169.3$ & $804.1 \pm 308.2$ & $<0.001$ \\
\hline ASPI & $579.5 \pm 238.6$ & $958.4 \pm 351.0$ & $1055.9 \pm 334.3$ & $793.8 \pm 209.2$ & $775.1 \pm 239.2$ & $667.8 \pm 287.1$ & $918.2 \pm 383.3$ & $<0.001$ \\
\hline TRAP & $1021.7 \pm 211.9$ & $1142.8 \pm 201.7$ & $1123.6 \pm 192.5$ & $1033.1 \pm 201.4$ & $1074.6 \pm 254.2$ & $1131.3 \pm 288.5$ & $1230.5 \pm 358.9$ & 0.022 \\
\hline EXTEM CT & $62.4 \pm 6.1$ & $70.5 \pm 15.2$ & $61.1 \pm 18.7$ & $61.4 \pm 7.0$ & $67.2 \pm 16.0$ & $66.6 \pm 9.6$ & $65.6 \pm 11.1$ & 0.074 \\
\hline EXTEM MCF & $63.7 \pm 4.5$ & $57.2 \pm 5.2$ & $60.3 \pm 5.8$ & $65.3 \pm 4.3$ & $66.5 \pm 3.7$ & $70.5 \pm 4.3$ & $70.6 \pm 3.5$ & $<0.001$ \\
\hline EXTEM A10 & $60.0 \pm 4.6$ & $51.4 \pm 5.1$ & $58.9 \pm 4.8$ & $60.7 \pm 4.8$ & $61.8 \pm 3.7$ & $66.4 \pm 4.1$ & $67.1 \pm 4.2$ & $<0.001$ \\
\hline $\begin{array}{l}\text { EXTEM } \\
\text { ALPHA } \\
\text { ANGLE }\end{array}$ & $77.7 \pm 2.2$ & $72.3 \pm 3.7$ & $76.8 \pm 2.3$ & $78.3 \pm 2.1$ & $73.0 \pm 18.5$ & $79.0 \pm 4.4$ & $80.1 \pm 2.3$ & 0.048 \\
\hline INTEM CT & $200.3 \pm 44.7$ & $216.1 \pm 57.1$ & $172.7 \pm 38.0$ & $191.7 \pm 43.9$ & $197.4 \pm 68.1$ & $176.6 \pm 33.4$ & $162.3 \pm 33.6$ & 0.001 \\
\hline INTEM MCF & $59.0 \pm 4.5$ & $54.4 \pm 5.4$ & $58.9 \pm 4.3$ & $61.3 \pm 5.2$ & $63.2 \pm 5.7$ & $68.2 \pm 4.5$ & $68.9 \pm 4.2$ & $<0.001$ \\
\hline INTEM A10 & $57.8 \pm 4.6$ & $50.5 \pm 5.2$ & $57.9 \pm 3.8$ & $59.5 \pm 4.2$ & $59.5 \pm 10.2$ & $65.8 \pm 4.0$ & $66.4 \pm 4.7$ & $<0.001$ \\
\hline $\begin{array}{l}\text { INTEM } \\
\text { ALPHA } \\
\text { ANGLE }\end{array}$ & $74.5 \pm 5.2$ & $71.4 \pm 5.1$ & $76.0 \pm 4.5$ & $76.8 \pm 3.5$ & $76.6 \pm 5.3$ & $79.3 \pm 1.8$ & $79.5 \pm 3.1$ & $<0.001$ \\
\hline FIBTEM CT & $54.3 \pm 12.2$ & $60.0 \pm 6.0$ & $53.6 \pm 6.0$ & $55.8 \pm 18.7$ & $57.8 \pm 7.1$ & $71.0 \pm 38.2$ & $61.7 \pm 9.4$ & 0.049 \\
\hline FIBTEM MCF & $21.0 \pm 5.1$ & $14.6 \pm 3.8$ & $22.2 \pm 4.4$ & $29.4 \pm 4.5$ & $30.1 \pm 3.7$ & $31.3 \pm 6.8$ & $32.5 \pm 6.1$ & $<0.001$ \\
\hline FIBTEM A10 & $20.8 \pm 5.0$ & $14.0 \pm 3.8$ & $21.6 \pm 4.3$ & $28.8 \pm 4.6$ & $28.9 \pm 3.4$ & $30.3 \pm 6.4$ & $31.4 \pm 5.9$ & $<0.001$ \\
\hline $\begin{array}{l}\text { FIBTEM } \\
\text { ALPHA } \\
\text { ANGLE }\end{array}$ & $76.0 \pm 5.6$ & $69.8 \pm 11.9$ & $77.2 \pm 3.4$ & $76.9 \pm 5.1$ & $78.6 \pm 3.4$ & $79.6 \pm 2.9$ & $80.4 \pm 2.0$ & $<0.001$ \\
\hline Troponini & $0.1 \pm 0.2$ & $3.4 \pm 2.4$ & $5.8 \pm 6.1$ & $3.9 \pm 4.8$ & $2.3 \pm 2.8$ & $1.2 \pm 1.7$ & $0.7 \pm 1.0$ & $<0.001$ \\
\hline CK MB & $21.7 \pm 9.4$ & $54.0 \pm 19.9$ & $54.5 \pm 37.8$ & $43.4 \pm 40.1$ & $34.4 \pm 28.6$ & $26.2 \pm 27.9$ & $22.7 \pm 24.6$ & $<0.001$ \\
\hline ACT & - & $130.0 \pm 15.0$ & $128.0 \pm 9.2$ & $127.7 \pm 7.3$ & $127.1 \pm 8.1$ & $125.2 \pm 8.3$ & $125.9 \pm 7.8$ & 0.044 \\
\hline
\end{tabular}

NOTE: values are displayed as arithmetic mean \pm SD

Table 4. Comparison of values in patients with and without bleeding

\begin{tabular}{|c|c|c|c|}
\hline Parameter & $\begin{array}{c}\text { Without bleeding } \\
(n=16)\end{array}$ & $\begin{array}{c}\text { With bleeding } \\
(n=12)\end{array}$ & $\mathbf{p}$ \\
\hline Age & $63.25 \pm 6.71$ & $65.33 \pm 7.14$ & 0.441 \\
\hline \multicolumn{4}{|l|}{ Gender } \\
\hline Male & $13(81.3 \%)$ & $9(75 \%)$ & 0.690 \\
\hline Female & $3(18.8 \%)$ & $3(25 \%)$ & \\
\hline Smoking & $3(18.8 \%)$ & $3(25 \%)$ & 0.690 \\
\hline Diabetes & $10(62.5 \%)$ & $3(25 \%)$ & 0.049 \\
\hline \multicolumn{4}{|l|}{ Regulation of DM } \\
\hline Without regulation & $0(0 \%)$ & $1(8.3 \%)$ & 0.053 \\
\hline Oral & $5(31.3 \%)$ & $1(8.3 \%)$ & \\
\hline Insulin & $5(31.3 \%)$ & $1(8.3 \%)$ & \\
\hline BMI & $31.43 \pm 4.44$ & $27.97 \pm 3.64$ & 0.032 \\
\hline Trigliceridi & $2.18 \pm 0.89$ & $1.44 \pm 0.57$ & 0.012 \\
\hline Cholesterol & $5.16 \pm 1.91$ & $3.29 \pm 1.96$ & 0.019 \\
\hline Urea & $5.75 \pm 1.14$ & $7.21 \pm 1.45$ & 0.009 \\
\hline Creatinine & $91.85 \pm 15.64$ & $100.76 \pm 21.05$ & 0.232 \\
\hline EF percents & $54.56 \pm 12.91$ & $52.92 \pm 7.22$ & 0.672 \\
\hline Type of surgery (number of grafts) & $2.56 \pm 0.51$ & $2.58 \pm 1.08$ & 0.952 \\
\hline Duration of EKK & $100.75 \pm 15.62$ & $104.5 \pm 29.51$ & 0.694 \\
\hline Clamping time & $43.56 \pm 8.66$ & $48.75 \pm 14.59$ & 0.289 \\
\hline The amount of given cardioplegia & $1196.88 \pm 315.95$ & $1108.33 \pm 222.42$ & 0.392 \\
\hline Defibrilation & $14(87.5 \%)$ & $12(100 \%)$ & 0.204 \\
\hline Diuresis & $1400 \pm 476.1$ & $1275 \pm 690.36$ & 0.597 \\
\hline ACT at admittance & $144.44 \pm 11.84$ & $131.67 \pm 11.97$ & 0.010 \\
\hline
\end{tabular}




\begin{tabular}{|c|c|c|c|}
\hline ACT after AH & $599.56 \pm 95.68$ & $564.33 \pm 116.39$ & 0.403 \\
\hline ACT after AP & $125.63 \pm 14.8$ & $126 \pm 8.14$ & 0.933 \\
\hline Autotransfusion & $578.44 \pm 121.2$ & $556.67 \pm 132.59$ & 0.660 \\
\hline Hospitalization in intensive unit & $4.5 \pm 1.32$ & $4.58 \pm 1.31$ & 0.869 \\
\hline Hospitalization in semi- intensive unit & $2.44 \pm 1.46$ & $2.42 \pm 1.38$ & 0.970 \\
\hline Hospitalization total & $6.94 \pm 0.57$ & $7 \pm 0.43$ & 0.744 \\
\hline WBC preoperatively & $7.3 \pm 1.39$ & $7.11 \pm 1.94$ & 0.775 \\
\hline RBC preoperatively & $4.58 \pm 0.39$ & $4.61 \pm 0.36$ & 0.841 \\
\hline HGB preoperatively & $141.75 \pm 8.92$ & $138.42 \pm 11.62$ & 0.418 \\
\hline HCT preoperatively & $38.59 \pm 10.49$ & $40.48 \pm 3.62$ & 0.512 \\
\hline PLT preoperatively & $269.31 \pm 74.43$ & $204.67 \pm 43.9$ & 0.008 \\
\hline MPV preoperatively & $8.61 \pm 0.71$ & $8.89 \pm 0.82$ & 0.357 \\
\hline CRP preoperatively & $4.38 \pm 3.51$ & $2.87 \pm 4.34$ & 0.334 \\
\hline P SEP preoperatively & $172.07 \pm 65.86$ & $184.92 \pm 50.38$ & 0.564 \\
\hline PT preoperatively & $11.46 \pm 1.22$ & $11.38 \pm 1.49$ & 0.892 \\
\hline INR preoperatively & $1.07 \pm 0.1$ & $1.07 \pm 0.13$ & 0.903 \\
\hline APTT preoperatively & $27.23 \pm 3.06$ & $25.25 \pm 3.31$ & 0.120 \\
\hline Fibrinogen preoperatively & $3.99 \pm 2.35$ & $4.23 \pm 1.32$ & 0.739 \\
\hline AT III preoperatively & $99.18 \pm 11.49$ & $102.41 \pm 17.36$ & 0.583 \\
\hline D dimer preoperatively & $244.38 \pm 122.48$ & $332.17 \pm 207.52$ & 0.210 \\
\hline ADP HS preoperatively & $449.75 \pm 199.9$ & $416.33 \pm 154.6$ & 0.622 \\
\hline ASPI preoperatively & $587.19 \pm 220.69$ & $569.25 \pm 270.47$ & 0.853 \\
\hline TRAP preoperatively & $962.38 \pm 216.98$ & $1100.83 \pm 184.52$ & 0.080 \\
\hline EXTEM CT preoperatively & $62.81 \pm 5.55$ & $61.83 \pm 6.95$ & 0.692 \\
\hline EXTEM MCF preoperatively & $64.25 \pm 5.27$ & $63 \pm 3.22$ & 0.446 \\
\hline EXTEM A10 preoperatively & $60.94 \pm 5.35$ & $58.83 \pm 3.16$ & 0.205 \\
\hline EXTEM ALPHA ANGLE preoperatively & $78.25 \pm 2.02$ & $76.92 \pm 2.28$ & 0.121 \\
\hline INTEM CT preoperatively & $187.25 \pm 39.77$ & $217.75 \pm 46.45$ & 0.081 \\
\hline INTEM MCF preoperatively & $59.88 \pm 4.52$ & $57.75 \pm 4.31$ & 0.218 \\
\hline INTEM A10 preoperatively & $58.75 \pm 4.7$ & $56.42 \pm 4.19$ & 0.178 \\
\hline INTEM ALPHA ANGLE preoperatively & $76.44 \pm 2.78$ & $71.92 \pm 6.59$ & 0.042 \\
\hline FIBTEM CT preoperatively & $57.56 \pm 5.53$ & $50 \pm 16.94$ & 0.161 \\
\hline FIBTEM MCF preoperatively & $22.38 \pm 5.57$ & $19.25 \pm 3.77$ & 0.089 \\
\hline FIBTEM A10 preoperatively & $21.81 \pm 5.62$ & $19.33 \pm 3.7$ & 0.172 \\
\hline FIBTEM ALPHA ANGLE preoperatively & $77.31 \pm 5.4$ & $74.33 \pm 5.69$ & 0.175 \\
\hline Troponines preoperatively & $0.01 \pm 0.03$ & $0.11 \pm 0.33$ & 0.350 \\
\hline CK MB preoperatively & $20.06 \pm 5.23$ & $23.87 \pm 13.14$ & 0.359 \\
\hline Drainage total & $875.06 \pm 313.4$ & $1110 \pm 448.46$ & 0.137 \\
\hline Diuresis total & $11718.13 \pm 4002.87$ & $11389.17 \pm 3253.59$ & 0.812 \\
\hline $\begin{array}{l}\text { WBC diffrence preoperatively and } 2 \text { h after } \\
\text { surgery }\end{array}$ & $2.07 \pm 3.31$ & $2.9 \pm 4.36$ & 0.587 \\
\hline $\begin{array}{l}\text { RBC difference preoperatively and } 2 \text { h after } \\
\text { surgery }\end{array}$ & $-0.35 \pm 0.47$ & $-0.39 \pm 0.39$ & 0.819 \\
\hline $\begin{array}{l}\text { HGB difference preoperatively and } 2 \text { h after } \\
\text { surgery }\end{array}$ & $-19.69 \pm 13.39$ & $-18.75 \pm 10.64$ & 0.838 \\
\hline $\begin{array}{l}\text { HCT difference preoperatively and } 2 \mathrm{~h} \text { after } \\
\text { surgery }\end{array}$ & $-2.41 \pm 10.84$ & $-7.65 \pm 8.99$ & 0.175 \\
\hline $\begin{array}{l}\text { PLT difference preoperatively and } 2 \mathrm{~h} \text { after } \\
\text { surgery }\end{array}$ & $-75.25 \pm 44.28$ & $-55.08 \pm 42.29$ & 0.233 \\
\hline $\begin{array}{l}\text { WBC difference } 2 \mathrm{~h} \text { after surgery and at the } \\
\text { end of follow-up }\end{array}$ & $-2.93 \pm 3.84$ & $-2.02 \pm 5.36$ & 0.624 \\
\hline $\begin{array}{l}\text { RBC difference } 2 h \text { after surgery and at the } \\
\text { end of follow-up }\end{array}$ & $-0.33 \pm 0.92$ & $-0.58 \pm 0.52$ & 0.371 \\
\hline $\begin{array}{l}\text { HGB difference } 2 \mathrm{~h} \text { after surgery and at the } \\
\text { end of a follow-up }\end{array}$ & $-13.44 \pm 16.65$ & $-14.67 \pm 15.16$ & 0.840 \\
\hline
\end{tabular}




\begin{tabular}{|c|c|c|c|}
\hline $\begin{array}{l}\text { HCT difference } 2 \mathrm{~h} \text { after surgery and at the } \\
\text { end of follow-up }\end{array}$ & $2.14 \pm 18.35$ & $-1.47 \pm 10.49$ & 0.518 \\
\hline $\begin{array}{l}\text { PLT difference } 2 \mathrm{~h} \text { after surgery and at the } \\
\text { end of follow-up }\end{array}$ & $105.26 \pm 119.96$ & $83.83 \pm 32.21$ & 0.504 \\
\hline $\begin{array}{l}\text { MPV difference preoperatively and at the } \\
\text { end of follow-up }\end{array}$ & $-1.14 \pm 0.71$ & $-0.93 \pm 0.95$ & 0.511 \\
\hline $\begin{array}{l}\text { CRP difference preoperatively and at the } \\
\text { end of follow up }\end{array}$ & $113.78 \pm 168.61$ & $87.08 \pm 68.8$ & 0.573 \\
\hline $\begin{array}{l}\text { P SEP difference preoperatively and at the } \\
\text { end of follow-up }\end{array}$ & $299.62 \pm 289.07$ & $362.42 \pm 307.98$ & 0.589 \\
\hline $\begin{array}{l}\text { PT difference preoperatively and at the end } \\
\text { of follow-up }\end{array}$ & $0.51 \pm 1.87$ & $2.54 \pm 5.31$ & 0.226 \\
\hline $\begin{array}{l}\text { INR difference preoperatively and at the } \\
\text { end of follow-up }\end{array}$ & $0.1 \pm 0.14$ & $0.26 \pm 0.51$ & 0.321 \\
\hline $\begin{array}{l}\text { APTT difference preoperatively and at the } \\
\text { end of follow-up }\end{array}$ & $-0.56 \pm 3.22$ & $3.3 \pm 4.35$ & 0.018 \\
\hline $\begin{array}{l}\text { Fibrinogen difference preoperatively and at } \\
\text { the end of follow-up }\end{array}$ & $4.94 \pm 3.26$ & $5.27 \pm 2.57$ & 0.762 \\
\hline $\begin{array}{l}\text { AT III difference preoperatively and at the } \\
\text { end of follow-up }\end{array}$ & $-1.01 \pm 23.4$ & $-8.83 \pm 22.11$ & 0.375 \\
\hline $\begin{array}{l}\text { D dimer difference preoperatively and at } \\
\text { the end of follow-up }\end{array}$ & $843.38 \pm 1076.16$ & $1602.92 \pm 1658.62$ & 0.184 \\
\hline $\begin{array}{l}\text { ADP HS difference preoperatively and at } \\
\text { the end of follow-up }\end{array}$ & $403.88 \pm 349.86$ & $321.67 \pm 356.48$ & 0.549 \\
\hline $\begin{array}{l}\text { ASPI difference preoperatively and at the } \\
\text { end of follow-up }\end{array}$ & $378.94 \pm 394.24$ & $285 \pm 496.68$ & 0.595 \\
\hline $\begin{array}{l}\text { TRAP difference preoperatively and at the } \\
\text { end of follow-up }\end{array}$ & $256.31 \pm 497.59$ & $145.42 \pm 338.83$ & 0.490 \\
\hline $\begin{array}{l}\text { EXTEM CT difference preoperatively and at } \\
\text { the end of follow-up }\end{array}$ & $1.63 \pm 12.96$ & $5.33 \pm 15.65$ & 0.512 \\
\hline $\begin{array}{l}\text { EXTEM MCF difference preoperatively and } \\
\text { at the end of follow-up }\end{array}$ & $6.5 \pm 4.82$ & $7.5 \pm 5.16$ & 0.607 \\
\hline $\begin{array}{l}\text { EXTEM A10 difference preoperatively and } \\
\text { at the end of follow-up }\end{array}$ & $6.31 \pm 5.1$ & $8.08 \pm 5.65$ & 0.401 \\
\hline $\begin{array}{l}\text { EXTEM ALPHA ANGLE difference } \\
\text { preoperatively and at the end of follow-up }\end{array}$ & $2.31 \pm 2.75$ & $2.67 \pm 3.7$ & 0.783 \\
\hline $\begin{array}{l}\text { INTEM CT difference preoperativelyand at } \\
\text { the end of follow-up }\end{array}$ & $-20.69 \pm 46.07$ & $-61.08 \pm 63.53$ & 0.078 \\
\hline $\begin{array}{l}\text { INTEM MCF difference preoperatively and } \\
\text { at the end of follow-up }\end{array}$ & $8.75 \pm 4.93$ & $11.42 \pm 7.37$ & 0.292 \\
\hline $\begin{array}{l}\text { INTEM A10 difference preoperatively and } \\
\text { at the end of follow-up }\end{array}$ & $7.38 \pm 5.2$ & $10.25 \pm 7.47$ & 0.268 \\
\hline $\begin{array}{l}\text { INTEM ALPHA ANGLE difference } \\
\text { preoperatively and at the end of follow-up }\end{array}$ & $3.06 \pm 2.59$ & $7.67 \pm 8.42$ & 0.091 \\
\hline $\begin{array}{l}\text { FIBTEM CT difference preoperatively and at } \\
\text { the end of follow-up }\end{array}$ & $3.19 \pm 9.93$ & $12.92 \pm 24.93$ & 0.223 \\
\hline $\begin{array}{l}\text { FIBTEM MCF difference preoperatively and } \\
\text { at the end of follow-up }\end{array}$ & $9.63 \pm 6.63$ & $13.83 \pm 6.9$ & 0.118 \\
\hline $\begin{array}{l}\text { FIBTEM A10 difference preoperatively and } \\
\text { at the end of follow up }\end{array}$ & $9.19 \pm 6.12$ & $12.67 \pm 7.02$ & 0.185 \\
\hline $\begin{array}{l}\text { FIBTEM ALPHA ANGLE difference } \\
\text { preoperatively and at the end of follow-up }\end{array}$ & $3.19 \pm 5.96$ & $6 \pm 5.56$ & 0.211 \\
\hline $\begin{array}{l}\text { Troponines- difference preoperatively and } \\
\text { at the end of follow-up }\end{array}$ & $0.64 \pm 0.87$ & $0.68 \pm 1.2$ & 0.910 \\
\hline $\begin{array}{l}\text { CK MB difference preoperatively and at the } \\
\text { end of follow-up }\end{array}$ & $-4.38 \pm 12.89$ & $8.06 \pm 22.02$ & 0.100 \\
\hline $\begin{array}{l}\text { ACT difference } 2 \mathrm{~h} \text { after surgery and at the } \\
\text { end of follow up }\end{array}$ & $-6 \pm 12.12$ & $-1.67 \pm 4.7$ & 0.206 \\
\hline
\end{tabular}

Multivariate logistic regression analysis, confirmed that the only factor significantly associated with the risk of bleeding, is a difference in APTT values preoperatively and at the end of the follow-up period (Table 5). An increase in this difference is associated with an increased risk of bleeding.
Variance analysis for repeated measurements showed that significant differences between the group of patients with and without bleeding existed at the PLT and ACT values over the entire period of follow-up (Table 6, Graph 1). 
Table 5. Association of the investigated parametres and risk for bleeding, results of multivariate logistic regression analysis

\begin{tabular}{|l|c|c|c||c||}
\hline \multicolumn{1}{|c|}{ Parameter } & \multirow{2}{*}{ OR } & \multicolumn{2}{c||}{$\begin{array}{c}\text { 95\% confidence } \\
\text { interval }\end{array}$} & P \\
\cline { 3 - 5 } & & Lower & Upper & \\
\hline \hline $\begin{array}{l}\text { APTT difference preoperatively and at he end of } \\
\text { follow-up }\end{array}$ & 2.87 & 1.141 & 7.22 & 0.025 \\
\hline Constant & 1421.1 & & & 0.023 \\
\hline
\end{tabular}

Table 6. Evaluation of the effects of the group (bleeding) and interaction between time and group on the values of individual parameters during the entire monitoring period, results of variance analysis for repeated measurements (RM ANOVA)

\begin{tabular}{|c|c|c|}
\hline Parameter & Bleeding & Time*bleeding \\
\hline WBC & 0.345 & 0.225 \\
\hline RBC & 0.381 & 0.540 \\
\hline HGB & 0.299 & 0.771 \\
\hline HCT & 0.746 & 0.263 \\
\hline PLT & 0.001 & 0.780 \\
\hline MPV & 0.117 & 0.270 \\
\hline CRP & 0.435 & 0.705 \\
\hline PSEP & 0.332 & 0.227 \\
\hline PT & 0.341 & 0.552 \\
\hline INR & 0.514 & 0.404 \\
\hline APTT & 0.479 & 0.439 \\
\hline Fibrinogen & 0.210 & 0.051 \\
\hline ATIII & 0.603 & 0.616 \\
\hline Ddimer & 0.118 & 0.288 \\
\hline ADPHS & 0.898 & 0.301 \\
\hline ASPI & 0.152 & 0.112 \\
\hline TRAP & 0.293 & 0.478 \\
\hline EXTEMCT & 0.240 & 0.181 \\
\hline EXTEMMCF & 0.170 & 0.160 \\
\hline EXTEMA10 & 0.080 & 0.682 \\
\hline EXTEMALPHAANGLE & 0.567 & 0.234 \\
\hline INTEMCT & 0.439 & 0.218 \\
\hline INTEMMCF & 0.120 & 0.299 \\
\hline INTEMA10 & 0.118 & 0.153 \\
\hline INTEMALPHAANGLE & 0.143 & 0.212 \\
\hline FIBTEMCT & 0.269 & 0.519 \\
\hline FIBTEMMCF & 0.834 & 0.227 \\
\hline FIBTEMA10 & 0.838 & 0.315 \\
\hline FIBTEMALPHAANGLE & 0.975 & 0.540 \\
\hline Troponines & 0.774 & 0.652 \\
\hline СКМВ & 0.136 & 0.139 \\
\hline ACT & $<0.001$ & 0.296 \\
\hline
\end{tabular}



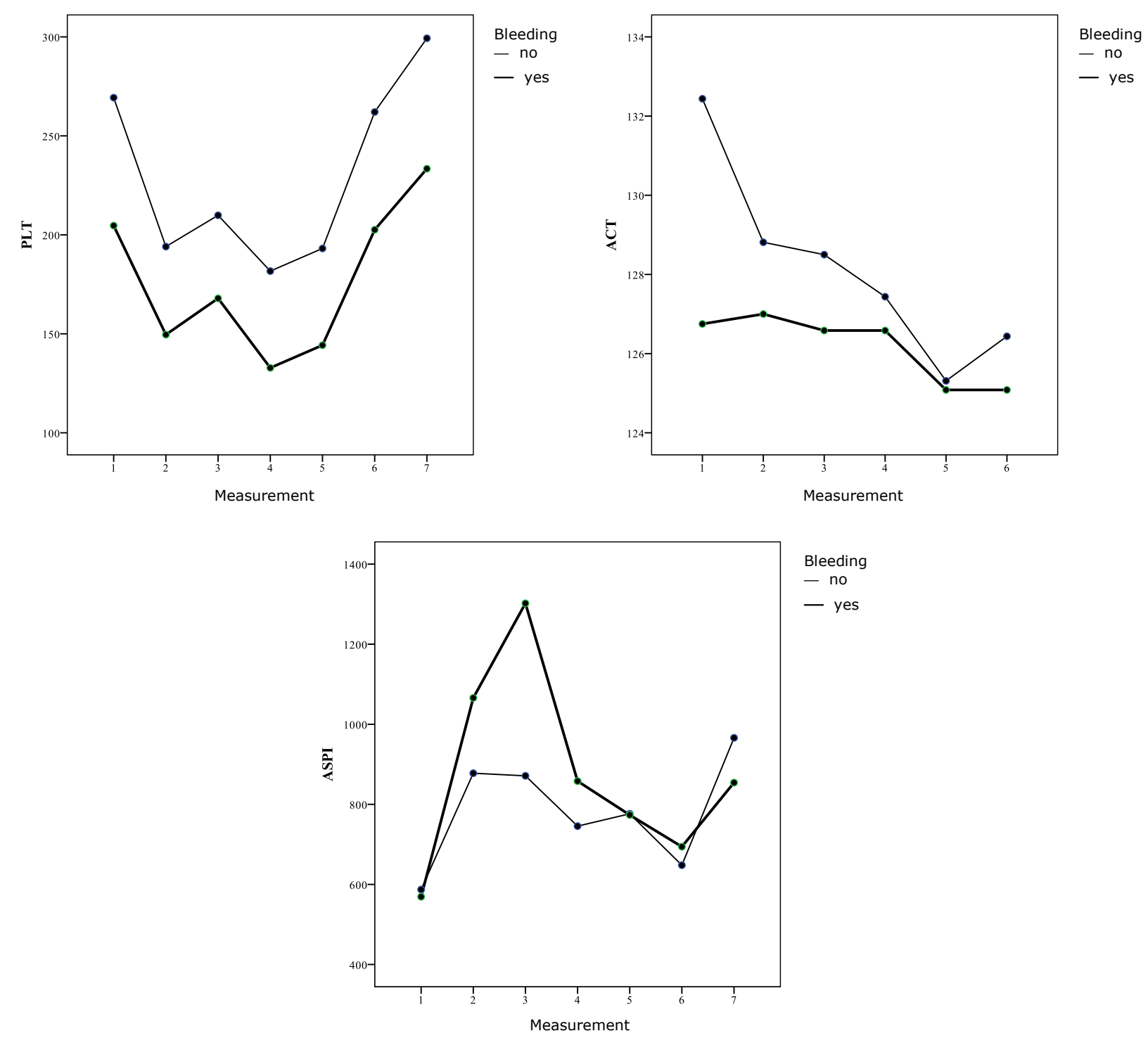

Graph 1. PLT, ACT, and ASPI values in patients with and without bleeding

Changes in ASPI values occurred in a significantly different way throughout the entire monitoring period in patients with and without bleeding.

There were no significant effects of the group (bleeding) and the interaction between time and group in the overall monitoring period regarding the value of other parameters.
Multivariate linear regression analysis showed that the only factor significantly associated with the change in the total allogeneic transfusion value was the value of urea (Table 7). Increase in urea levels is associated with an increase in total allogeneic blood transfusion values (Graph 2).

Table 7. Connection betweenl total allogeneic transfusion and values of other investigated features, results of multivariate linear regression analysis

\begin{tabular}{|c||c||c|c|c||}
\hline \multirow{2}{*}{ Parameter } & \multirow{2}{*}{ B } & \multicolumn{3}{|c|}{ 95\% confidence interval } \\
\cline { 3 - 5 } & & Lower & Upper & P \\
\hline \hline (Constant) & -887.191 & -1541.135 & -233.246 & 0.010 \\
\hline Urea & 178.383 & 78.292 & 278.474 & 0.001 \\
\hline
\end{tabular}




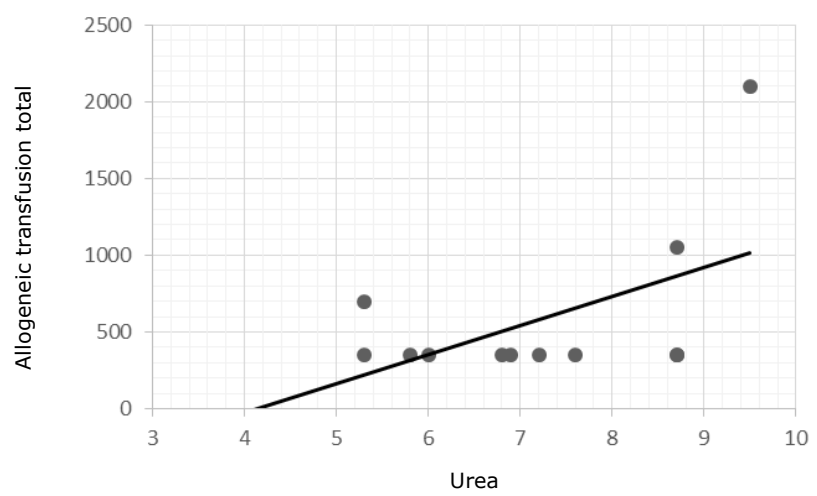

Graph 2. The relationship between the value of total allogeneic transfusion amount and urea values

Multivariate linear regression analysis confirmed: the type of surgery (number of grafts), diuresis, ACT after AP, and an increase in HCT from preoperative period to period $2 \mathrm{~h}$ after surgery, as factors significantly associated with changes in the number of days in intensive care (Table 8).

An increase in the number of grafts and diuresis is associated with an increase in the number of days in intensive care, while the rise in ACT after AP and HCT difference preoperatively and $2 \mathrm{~h}$ after surgery, are associated with a decrease in the number of days in intensive care (Graph 3).

Multivariate linear regression analysis, confirmed the amount of cardioplegia, the difference in RBC values in the period of 2 hours after the operation to the end of the monitoring period, and the difference in values of PT and EXTEM CT in the period before surgery to the end monitoring period as factors sig- nificantly associated with changes in the number of days of total hospitalization (Table 9).

An increase in the amount of cardioplegia has been associated with a reduction in the number of days of total hospitalization, while an increase in RBC values in the period from 2 hours after operation to the end of the follow-up period, as well as an increase in the values of PT and EXTEM CT from the period before surgery to the end of the monitoring period, has been associated to increased number of days of total hospitalization.

Multivariate linear regression analysis, confirmed the preoperative value of INR as the only factor significantly associated with changes in total drainage value (Table 10). The increase in preoperative INR value is associated with an increase in the total drainage value (Graph 4).

Table 8. The relationship between the number of days in intensive care and the value of all other investigated features, the results of the multivariate linear regression analysis

\begin{tabular}{|l|r|rrr||}
\hline \multicolumn{1}{|c|}{ Parameters } & \multirow{2}{*}{ B } & 95\% confidence interval \\
\cline { 3 - 5 } & & Lower & Upper & \multicolumn{1}{c|}{ p } \\
\hline \hline (Constant) & 6.722 & 3.410 & 10.034 & $<0.001$ \\
\hline Type of surgery (number of grafts) & 0.674 & 0.310 & 1.038 & 0.001 \\
\hline ACT after AP & -0.042 & -0.065 & -0.019 & 0.001 \\
\hline HCT difference preoperatively and 2h after surgery & -0.059 & -0.087 & -0.031 & $<0.001$ \\
\hline Diuresis & 0.001 & $<0.001$ & 0.001 & 0.004 \\
\hline \hline
\end{tabular}



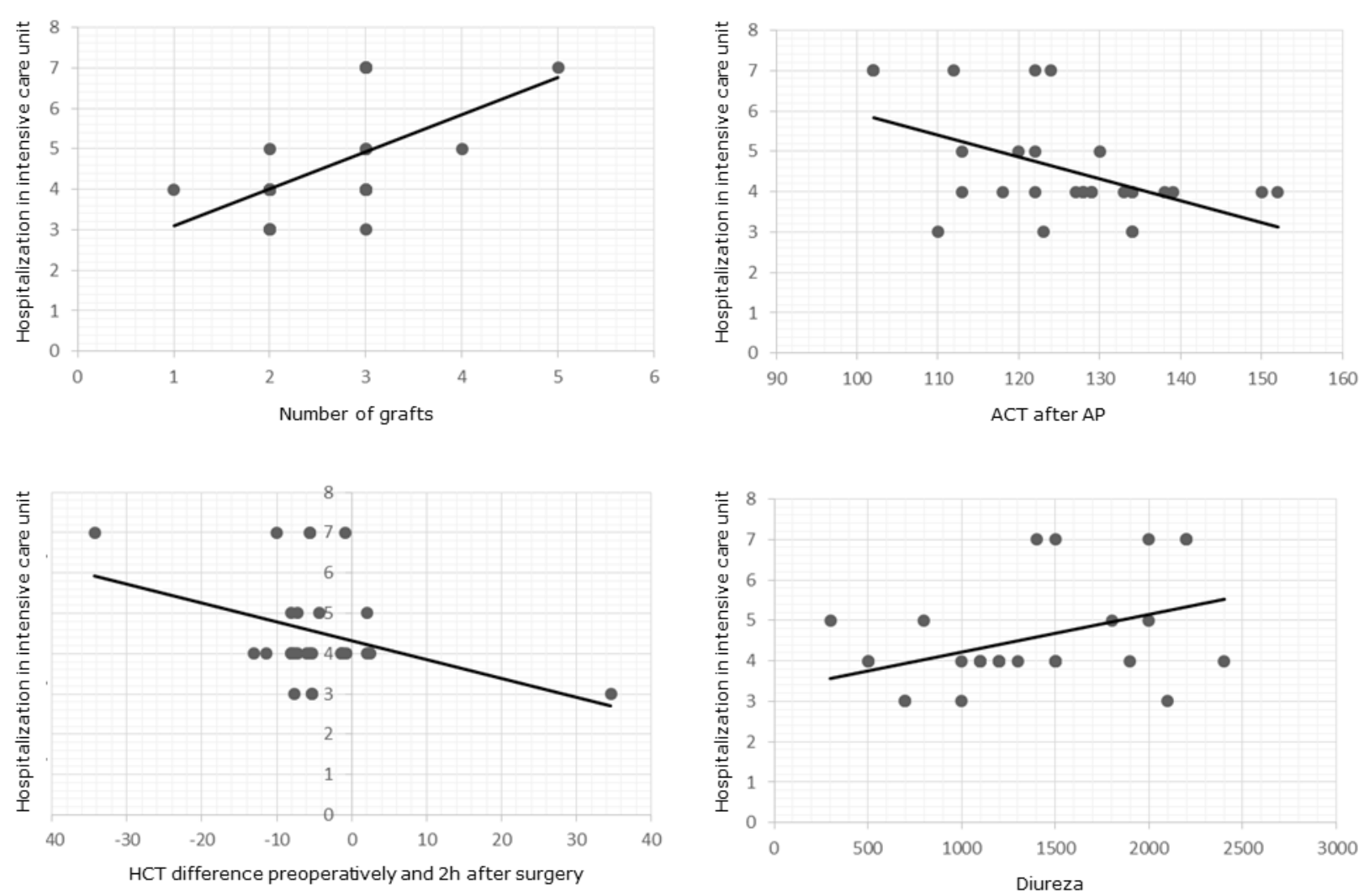

Graph 3. The relationship between the number of days in intensive care and the number of grafts, ACT after AP, HCT difference preoperatively and $2 \mathrm{~h}$ after surgery and diuresis

Table 9. The relationship between the number of days of total hospitalization and the value of all other investigated features, the results of multivariate linear regression analysis

\begin{tabular}{|c|c|c|c|c|}
\hline \multirow{2}{*}{ Parameter } & \multirow{2}{*}{ B } & \multicolumn{3}{|c|}{$95 \%$ confidence interval } \\
\hline & & Lower & Upper & $\mathbf{p}$ \\
\hline (Constant) & 7.786 & 7.302 & 8.270 & $<0.001$ \\
\hline The amount of given cardioplegia & -0.001 & -0.0012 & -0.0008 & 0.002 \\
\hline RBC diffrence $2 \mathrm{~h}$ after surgery and at the end of follow-up & 0.260 & 0.106 & 0.413 & 0.002 \\
\hline EXTEM CT difference preoperatively and at the end of follow-up & 0.016 & 0.008 & 0.024 & 0.001 \\
\hline PT difference preoperatively and at the end of follow-up & 0.035 & 0.004 & 0.067 & 0.030 \\
\hline
\end{tabular}

Table 10. The relationship between the total drainage value and the value of all other investigated features, the results of the multivariate linear regression analysis

\begin{tabular}{||l||c||ccc||}
\hline \multirow{2}{*}{ Parameter } & \multirow{2}{*}{ B } & \multicolumn{3}{c|}{ 95\% confidence interval } \\
\cline { 3 - 6 } & & Lower & Upper & p \\
\hline \hline (Constant) & 3052.12 & 1817.42 & 4286.82 & $<0.001$ \\
\hline INR preoperatively & -1942.87 & -3092.06 & -793.67 & 0.002 \\
\hline
\end{tabular}




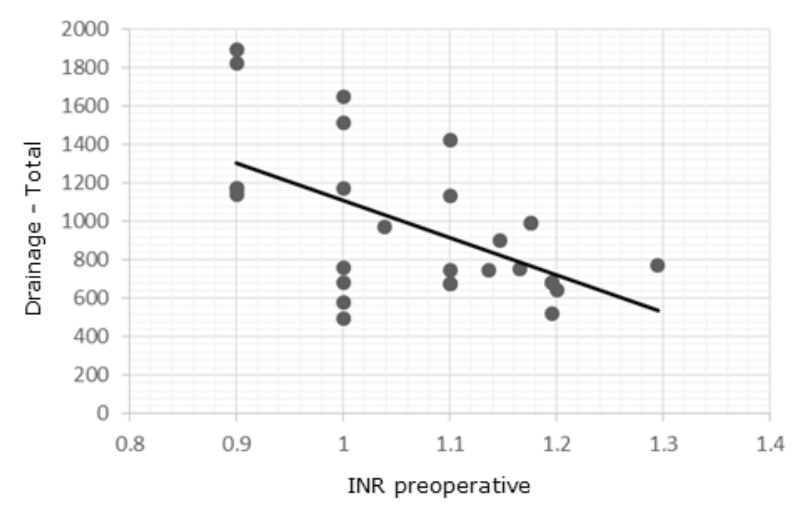

Graph 4. The relationship between total drainage and preoperative value of INR

\section{Discussion}

Cardiosurgical interventions carry a certain risk with them. This risk depends on age, gender, heart function, condition of the whole organism, urgency of the intervention, etc. Statistical analysis of a large number of cardio-surgical patients has determined the factors that influence the operational risk.

According to the National Blood Collection \& Utilization Survey (NBCUS), blood transfusions and blood derivatives, with the exception of platelet transfusions, declined between 2008. and 2011. in the United States (9). In this period, a reduction rate of $8.2 \%$ of red blood cell transfusions (RBC) was recorded, as well as a reduction in plasma transfusion by $13.4 \%$. In the United States, contrary to the general trend, there has been an increase in blood transfusions and blood derivatives during cardiovascular surgery during 2010, when a total of $34 \%$ of patients undergoing transfusion received red blood cells or other blood derivatives (10). Moreover, cardiac surgery was a branch of surgery that consumed the largest amounts of blood, just before orthopedic surgery $(11,12)$. Transfusion is often necessary during cardiovascular surgery to cure coagulopathy, blood loss, and hemodilution due to priming (13). Very often, patients who undergo cardiac surgery have numerous comorbidities such as anemia or myocardial infarction, which increase the risk of complications, and therefore the need for blood transfusion is higher $(10,11,14)$.

This study included a total of 28 patients who underwent surgical revascularization of the myocardium at the Clinic for Cardiovascular with Transplantation Surgery, Clinical Centre of Niš, 22 men (79\%) and 6 women $(21 \%)$, with average age of 64 . The most common risk factor for these patients was diabetes mellitus, which was present in $46.4 \%$ of patients. In our study, there was $21.4 \%$ smokers fewer than in other published studies (13-14). This study also showed that BMI was a significant indicator of the incidence of coronary disease, so the average BMI for examiners in our study was 29.94 .
The most commonly performed procedure for these patients was double and triple aorto-coronary bypass, with $46.4 \%$ of patients having a double aorto-coronary bypass, and $42.9 \%$ of patients with triple aorto-coronary bypass. The average number of grafts that patients received was 2.57 .

The average ECC time was 102 minutes and the time for the aortic cross clamp was 45 minutes, on average, $1158 \mathrm{ml}$ of cardioplegia was given.

Allogeneic transfusion was administered in 3 patients on the first postoperative day, and the average blood amount was $933.3 \mathrm{ml}$. On the second day it was applied in two patients, $350 \mathrm{ml}$ each, the third day in one patient, $700 \mathrm{ml}$, the fourth day in 4 patients, $350 \mathrm{ml}$ each.

On the fifth postoperative day allogeneic transfusion was not applied to all patients. On the sixth and seventh day, allogeneic transfusion was administered in 2 patients, in $350 \mathrm{ml}$ each. In total, allogeneic transfusion was received by 12 patients, and the approximate amount of blood delivered to them was $583 \mathrm{ml}$.

The diuresis was measured for the first three days in all 28 patients, and the average value was $2779 \mathrm{ml}, 3004 \mathrm{ml}$ and $2795 \mathrm{ml}$, by day. On the fourth day the diuresis was measured in 17 patients, on average $2852 \mathrm{ml}$, on the fifth day in 9 patients on average $3105 \mathrm{ml}$, the six in 2 patients at $2850 \mathrm{ml}$, and on the seventh day in one patient $1800 \mathrm{ml}$.

Platelets were administered only on the first day, in 4 patients with an average of 6.25 units, plasma, cryoprecipitate, volume, and crystalloids were not applied.

Several studies published the results and effects of blood transfusion in anemic and non-anemic, haemodynamically stable patients. (15-26). A retrospective study carried out in Cleveland Clinic showed that patients undergoing cardio-pulmonary bypass and who did not have anemia during the ECC procedure (hematocrit $>25 \%$ ), but who received an intraoperative blood, had a need for longer use of mechanical ventilation and they had reduced longterm survival compared to a non-anemic group of patients or with patients who had anemia but did not receive blood (27). Blood transfusions in patients un- 
dergoing surgical myocardial revascularization, who were preoperatively classified in a group with a low or moderate risk according to EuroSCORE $(<8)$ and who had a postoperative hemoglobin greater than $10 \mathrm{~g} \mathrm{/} \mathrm{dL}$ and a minimum postoperative blood loss and without postoperative complications in the first 24 hours of surgery, caused an increased risk of postoperative events and infections in relation to those patients who did not receive blood replacement $(17,21-23,28)$.

Shaw et al. compared the effects of blood transfusion in a group of patients who were stratified by the value of hematocrit. Preoperative hematocrit values were compared in patients undergoing cardiac surgery depending on whether they received blood transfusion or not (29). This study showed a statistically significantly higher rate of mortality after 30 days, for that group of patients who received blood transfusion compared to a group of patients who did not receive blood. These results are in agreement with previously published studies that confirm that there is a higher mortality rate in patients receiving blood transfusion (28, 30-33).

A retrospective study published by Schwann et al. investigated the correlation between blood transfusion and mortality in 6,947 patients who underwent surgical myocardial revascularization (34). The overall rate of the red blood cells transfusions was $33.9 \%$. Postoperative complications were present in $35.2 \%$ of patients, and this was statistically significantly higher in comparison to a group of patients who did not receive blood transfusion. The most common complications of this type were present in older women with comorbidities. Early mortality (30 days after surgery) and five-year survival were higher among the group of patients receiving blood transfusion, compared to those who did not receive red blood cells. The authors concluded that red blood cell transfusion increased the risk of cardiac and noncardiac mortality in patients who underwent surgical myocardial revascularization (34-36).

The initial decline after the operation, with a subsequent increase at the end of the monitoring period, was recorded for values of RBC, HGB, PLT, AT III, EXTEM MCF, EXTEM A10, EXTEM ALPHA ANGLE, INTEM MCF, INTEM A10, INTEM ALPHA ANGLE, FIBTEM MCF, FIBTEM A10 , FIBTEM ALPHA ANGLE and ACT.

The initial increase after surgery, with subsequent decline at the end of the monitoring period, was recorded for the values of WBC, CRP, fibrinogen, INTEM CT, troponins and CK MB.

The increasing trend during the entire monitoring period was recorded for values of $P$ SEP, $D$ dimer, ADP HS, ASPI, TRAP and FIBTEM CT.

In relation to preoperative values, the number of WBC significantly increased 2 hours after surgery, while the values of RBC, HGB, HCT, and PLT significantly dropped in the same period.

Diabetes was significantly more common in patients without bleeding. In patients without bleeding, significantly more BMI, triglyceride, cholesterol, preoperative ACT, PLT and INTEM ALPHA ANGLE were preoperatively detected, compare to patients with bleeding.
In patients with bleeding, significantly higher values of the urea and APTT difference preoperatively and at the end of the follow-up period were detected compared to patients without bleeding. The values of all other parameters did not differ significantly in patients with and without bleeding.

The multivariate logistic regression analysis, confirmed that the only factor significantly associated with the risk of bleeding, is a difference in APTT values preoperatively and at the end of the follow-up period. An increase in this difference is associated with an increased risk of bleeding.

The variance analysis for repeated measurements showed that significant differences between the group of patients with and without bleeding existed at the PLT and ACT values.

The changes in ASPI values occurred in a significantly different way throughout the entire monitoring period in patients with and without bleeding.

There were no significant effects of the group (bleeding) and the interaction between time and group in the overall monitoring period, in the value of other features.

The correlation analysis showed that the increased values of total allogeneic transfusion were significantly associated with elevated urea values and increased differences in APTT and CK MB values preoperatively and at the end of the follow-up period.

The multivariate linear regression analysis confirmed the value of urea as the only factor significantly associated with the change in the total allogeneic transfusion value. The increase in urea levels is associated with an increase in the total amount of allogeneic transfusion.

The correlation analysis showed that the increased number of days in intensive care was significantly associated with female gender, higher number of grafts, prolonged ECC time, aortic cross clamp time, diuresis, AT III preoperative, EXTEM CT preoperatively, increased differences in HCT, PT, INR preoperatively and at the end of the monitoring period.

An increased number of total hospitalization days was significantly associated with lowered values of given cardioplegia and PLT preoperative values with declines in WBC and HGB values in the period before surgery to 2 hours after surgery, as well as with the decline in troponine levels in the period before surgery to the end of the follow-up period.

The multivariate linear regression analysis confirmed the amount of cardioplegia, the difference in RBC values in the period of 2 hours after the operation to the end of the monitoring period, and the difference in values of PT and EXTEM CT in the period before surgery to the end of monitoring period as factors significantly associated with changes in the number of days of total hospitalization.

An increase in the amount of cardioplegia has been associated with a reduction in the number of days of the hospitalization, while an increase in RBC values in the period of 2 hours after operation to the end of the follow-up period, as well as an increase in the values of PT and EXTEM CT in the period before surgery to the end of the monitoring period, has been associated with increased number of hospitalization days overall. 
The correlation analysis showed that an increase in the total drainage value was significantly associated with an increase in triglyceride and preoperative HCT values, while the increase in total drainage value was significantly associated with a fall in preoperative INR. The multivariate linear regression analysis confirmed the preoperative value of INR as the only factor significantly associated with changes in total drainage value. The increase in preoperative INR value is associated with an increase in the total drainage value. A major dilemma in bleeding patients is whether coagulopathy or mechanical bleeding is the cause of the increase drainage and whether it is necessary to continue with the administration of hemostasic therapy and blood products or perform revision in the operating room. In less than ten minutes, ROTEM test guide doctors in which direction they should work.

The number of transfused allogeneic blood products is declining year after year, which points to the importance of modern monitoring in the indication of blood transfusions by the doctors. Transfusion of fresh plasma is also reduced. Transfusion of concentrated platelets remained at the same level, but percentage of cryoprecipitate transfusions increased year after year (37).

Despite improvements made with existing new techniques, most surgeons tend to accept a significant amount of blood loss as a feature of cardiac surgery (38). It is important to ensure adequate drainage and removal of blood from pericardium and pleura (it has high fibrinolytic activity and tissue factor of coagulation). Removing this blood and clot probably not only reduces the chance of excessive blood loss by preventing systemic coagulopathy, but it is likely to have beneficial effects on several other factors associated with surgery such as, for example, inflammation, atrial fibrillation, pericardial effusion (tamponade), and development of the adhesions (39). After careful evaluation, hemodilution appears to be the most pronounced factor associated with the development of coagulopathy after cardiac surgery, and probably plays an important role in the occurrence of blood loss after heart surgery (40). Fibrinogen is one of the most important factors in coagulation and it is possible that the clotting process falls below the critical level during hemodilution and therefore care should be taken and, if necessary administration of fibrinogen concentrate initiated (41).

Fibrinogen is an acute phase protein the level of which gradually increases during and after surgery in response to a surgical trauma and the use of ECC. The increased concentration of D-dimer and prothrombin fragment $1+2$ together with increased thrombin production indicate that the hypercoagulable state develops up to 5 days after cardiac surgery. The combination of these two factors (hypercoagulable state and the use of fibrinogen concentrates) can increase the risk of thromboembolic complications in the postoperative period. Therefore, adequate anticoagulant and (or) antiaggregation therapy to prevent the occurrence of thromboembolic complications in the postoperative course is necessary, especially in patients who do not receive vitamin $\mathrm{K}$ antagonists, even in patients without prior bleeding. A delicate balance between bleeding and hypercoagulable states must be maintained. The use of POC evaluation can provide a quicker and more complete insight into this delicate balance, creating more individualized treatment oriented to each patient in particular. A large variation in the patient's sensitivity to the use of clopidogrel often results in very different individual results before surgery, which requires further use and determination of the POC before, during and after cardiac surgery. An individual approach oriented to each patient can contribute to the reduction of perioperative and postoperative blood loss and minimizes the need for transfusion to a minimum (42).

Transfusion of red blood cells is common in cardiac surgery. The percentage of patients receiving blood transfusion during the perioperative period varies in literature: from 95\%, up to 10 years ago, to $49 \%$ of CABG patients over the past few years. Blood transfusion can improve systemic transport and distribution of oxygen, relieve regulation of vasomotor response, improve delivery of oxygen to the myocardium. On the other hand, there are data in the literature that indicate that transfusion damage is probably more serious than it has been valued so far and that blood transfusions are used more often than necessary (43). Even transfusion of one blood unit is associated with a significant risk of serious postoperative morbidity, the immediate goal should be to avoid transfusion whenever possible and not to apply it just to treat low hemoglobin levels, which is a common practice in over $50 \%$ of all patients receiving transfusion. Traditional concern over transfusion of blood and blood derivatives is reduced to the possibility of transmitting viral and bacterial infections or the occurrence of haemolytic reactions, which is rarely occurring. However, immunosuppression, lung damage, or dysfunction of the organs can occur with each recipient. Recently, Cleveland Clinic investigators have found that the administration of erythrocytes that have been stored for a longer period (> 14 days) is independently associated with an increased risk of complications and increases the estimated risk of death (30). This happens because the erythrocytes over time develop lesions and release cytokines, cell membranes fragment and release hemoglobin and free oxygen radicals. Obviously, there is a challenge to determine the circumstances in which transfusion is used. Unfortunately, the existing evidence is scarce, and the existing guidelines and recommendations are based on a low level of evidence. Assuring doctors to change their practice is not an easy task and an appropriate clinical assessment is used as a justification for transfusion.

Numerous strategies exist to minimize the need for a transfusion of blood and blood products in the perioperative period.

Some of the existing guidelines recommend: discontinuation of preoperative application of antithrombotic drugs, applying a restrictive attitude about the level of hemoglobin requiring red blood cell transfusion, the application of intraoperative blood savage techniques, and offpump CABG as one of the of surgical techniques that can reduce bleeding in the postoperative course. The study we conducted showed that using the POC and Rotem methods in the 
preoperative and postoperative period, can reduce postoperative bleeding to an acceptable level that in most patients does not require the use of blood and blood derivative transfusion. Antiagregation therapy was stopped before surgery and was re-administered after taking drains out. Reinfusion of the remaining blood at the end of the CPB and the use of the Cell Saver system were applied to all patients involved in our study. But perhaps most importantly, doctors should be encouraged to use a restrictive hemoglobin compensation model, which is also recommended in special guidelines: patients should be given blood transfusions when hemoglobin is less than $7 \mathrm{~g}$ / $\mathrm{dL}$, where transfusion is not indicated for improving oxygen transport and when the hemoglobin concentration was higher than $10 \mathrm{~g} / \mathrm{dL}$. About $20 \%$ of all CABG operations are in off-pump technique. The use of $C P B$ during $C A B G$ is associated with more harmful effects, including hemodilution, activation of coagulation factors, and a decrease in the number and function of platelets, leading to coagulopathy that can lead to extensive bleeding and the need for massive blood transfusions. Because of this, off-pump procedures would be expected to lead to a reduction in the incidence of postoperative complications, and are also recommended to reduce the need for transfusion. However, in our study, we did not notice the significant differences between individual patient groups in whom increased bleeding could be expected in the intra and postoperative period. The number of platelets and their function was tracked at 8 different time points. Our study did not show that there was statistically significant correlation between individual groups of patients compared to basic biochemical and inflammatory parameters with the number and function of platelets. There has been a decrease in platelet counts intraoperatively, and a gradual increase in their number in the immediate postoperative period. Increased perioperative bleeding was associated only with a reduced number and platelet function as well as elevated ACT rates.

The only parameter in our study that was associated with increased postoperative drainage was increased preoperative value of INR. In our study, only 4 patients $(14.28 \%)$ received platelets while 12 patients received a blood supply (42\%), an average of $583 \mathrm{ml}$. We didn't have any reintervention because of bleeding. Our study could not show independent prognostic factors of greater postoperative drainage compared to the classic operational parameters that are being monitored (aortic cross-clamp and ECC time...), nor in relation to the parameters of inflammation.

\section{Conclusion}

Based on the conducted research and the obtained results, it can be concluded:

- Surgical myocardial revascularization is a safe technique with a minimum morbidity rate.

- By using of modern methods for preoperative hemostasis monitoring (Multiplate, Rotem), the risk of postoperative bleeding can be significantly reduced, as well as the need for transfusion of red blood cells and other blood derivatives.

- The only preoperative independent prognostic parameter for increased postoperative drainage was INR.

- ACT was an independent postoperative prognostic parameter of increased postoperative drainage, probably due to delayed or prolonged heparin activity

- Inflammation parameters did not show association with the occurrence of postoperative complications.

- Diabetes was significantly more common in patients without bleeding. The same conclusion was for patients with higher BMI.

- In patients with bleeding significantly higher values of urea and the difference in APTT preoperatively and at the end of the follow-up period were detected compared to patients without bleeding.

- Multivariate logistic regression analysis confirmed the difference in APTT values preoperatively and at the end of the monitoring period, as the only factor significantly associated with the risk of bleeding.

- Correlation analysis showed that the increased values of total allogeneic transfusion were significantly associated with the elevated values of urea and increased differences in APTT and CK MB values preoperatively and at the end of the follow-up period.

- Multivariate linear regression analysis confirmed the urea value as the only factor significantly associated with the change in the total transfusion value. The increase in urea levels is associated with the increase in the total amount of allogeneic transfusion.

- The correlation analysis showed that the increased number of days in the intensive care was significantly associated with female gender, high number of grafts and prolonged ECC and aortic cross -clamp time, HCT, PT, and INR values preoperatively and at the end of the follow-up period. 


\section{References}

1. De Backer G. Epidemiology and prevention of cardiovascular disease: Quo vadis? Eur J Prev Cardiol 2017; 24(7): 768-72. [CrossRef][PubMed]

2. Nichols M, Townsend N, Scarborough $P$, Rayner $M$. Cardiovascular disease in Europe 2014: epidemiological update. Eur Heart J 2014; 35(42): 2929. [CrossRef][PubMed]

3. Fuster V, Kelly BB, editors. Promoting Cardiovascular Health in the Developing World: A Critical Challenge to Achieve Global Health. Washington (DC): National Academies Press (US); 2010.

4. Incidencija i mortalitet od akutnog koronarnog sindroma u 2006, 2007, 2008, 2009, Srbija. Institut za javno zdravlje republike Srbije "Dr Milan Jovanović Batut"; Available from: http://www.batut.org.rs.

5. Wijns W, Kolh P, Danchin N, Di Mario C, Falk V, Folliguet $\mathrm{T}$, et al. Guidelines on myocardialrevascularization: The Task Force on Myocardial Revascularization of the European Society of Cardiology (ESC) and the European Association for Cardio-Thoracic Surgery (EACTS). Eur Heart J 2010; 31(20): 2501-55. [CrossRef][PubMed]

6. Herzog CA, Ma JZ, Collins AJ. Comparative survival of dialysis patients in the United Statesafter coronary angioplasty, coronary artery stenting, and coronary artery bypass surgery andimpact of diabetes. Circulation 2002; 106(17): 2207-11. [CrossRef][PubMed]

7. Fox K, Garcia MA, Ardissino D, Buszman P, Camici PG, Crea $F$, et al. Guidelines on themanagement of stable angina pectoris: executive summary: The Task Force on the Management of Stable Angina Pectoris of the European Society of Cardiology. Eur Heart J 2006; 27(11): 1341-81. [CrossRef][PubMed]

8. Trullàs JC, González-Franco Á. Major developments in the 2016 european guidelines for heart failure. Rev Clin Esp 2017; 217(7): 405-9. [CrossRef][PubMed]

9. US Department of Health and Human Services: The 2011 national blood collection and utilization survey report. Washington, DC: US Department of Health and Human Services, Office of the Assistant Secretary for Health. 2011; 15 Available from:

https://www.aabb.org/research/hemovigilance/bloods urvey/Documents/11-nbcus-report.pdf

10. Robich MP, Koch CG, Johnston DR, Schiltz N, Chandran Pillai A, Hussain ST, et al. Trends in blood utilization in United States cardiac surgical patients. Transfusion 2015; 55(4): 805-14. [CrossRef][PubMed]

11. Geissler RG, Rotering $H$, Buddendick $H$, Franz D, Bunzemeier $\mathrm{H}$, Roeder $\mathrm{N}$, et al. Utilisation of blood components in cardiac surgery: a single-centre retrospective analysis with regard to diagnosis-related procedures. Transfus Med Hemother 2015; 42(2): 75-82. [CrossRef][PubMed]

12. Stoicea N, Bergese SD, Ackermann W, Moran KR, Hamilton C, Joseph $N$, et al. Current status of blood transfusion and antifibrinolytic therapy in orthopedic surgeries. Front Surg 2015; 2:3. [CrossRef][PubMed]

13. Koch CG. Tolerating anemia: taking aim at the right target before pulling the transfusion trigger. Transfusion 2014;54(10 Pt 2):2595-7. [CrossRef][PubMed]

14. Ad N, Massimiano PS, Burton NA, Halpin L, Pritchard G, Shuman DJ, et al. Effect of patient age on blood product transfusion after cardiac surgery. J Thorac Cardiovasc Surg 2015; 150(1): 209-14.

[CrossRef][PubMed]
15. Vincent JL, Baron JF, Reinhart K, Gattinoni L, Thijs L, Webb $A$, et al. Anemia and blood transfusion in critically ill patients. JAMA 2002; 288(12): 1499-507. [CrossRef][PubMed]

16. Anía BJ, Suman VJ, Fairbanks VF, Rademacher DM, Melton $\sqcup$ 3rd.. Incidence of anemia in older people: an epidemiologic study in a well defined population. J Am Geriatr Soc 1997; 45(7): 825-31. [CrossRef] [PubMed]

17. Dejam A, Malley BE, Feng M, Cismondi F, Park S, Samani $S$, et al. The effect of age and clinical circumstances on the outcome of red blood cell transfusion in critically ill patients. Crit Care 2014; 18(4): 487. [CrossRef][PubMed]

18. Rodriguez RM, Corwin $\mathrm{HL}$, Gettinger A, Corwin MJ, Gubler D, Pearl RG. Nutritional deficiencies and blunted erythropoietin response as causes of the anemia of critical illness. J Crit Care 2001; 16(1): 36-41. [CrossRef][PubMed]

19. Corwin HL, Parsonnet KC, Gettinger A. RBC transfusion in the ICU. Is there a reason? Chest 1995; 108(3): 767-71. [CrossRef][PubMed]

20. Littenberg B, Corwin H, Gettinger A, Leichter J, Aubuchon J. A practice guideline and decision aid for blood transfusion.Immunohematology 1995; 11(3): 88-94. [PubMed]

21. Lako A, Bilali S, Memishaj S, Daka A, Dedej T, Nurka $T$, et al. The impact of blood use on patients undergoing coronary artery bypass surgery: a prospective study. G Chir 2014; 35(1-2): 20-6. [CrossRef][PubMed]

22. Westenbrink BD, Kleijn L, de Boer RA, Tijssen JG, Warnica WJ, Baillot R, et al. Sustained postoperative anaemia is associated with an impaired outcome after coronary artery bypass graft surgery: insights from the IMAGINE trial. Heart 2011; 97(19): 1590-6. [CrossRef][PubMed]

23. Patel NN, Avlonitis VS, Jones $\mathrm{HE}$, Reeves BC, Sterne JA, Murphy GJ. Indications for red blood cell transfusion in cardiac surgery: a systematic review and meta-analysis. Lancet Haematol 2015; 2(12): e54353. [CrossRef][PubMed]

24. Murphy GJ, Reeves BC, Rogers CA, Rizvi SI, Culliford $\mathrm{L}$, Angelini GD. Increased mortality, postoperative morbidity, and cost after red blood cell transfusion in patients having cardiac surgery. Circulation 2007; 116 (22): 2544-52. [CrossRef][PubMed]

25. Litmathe J, Boeken U, Feindt P, Gams E. Predictors of homologous blood transfusion for patients undergoing open heart surgery. Thorac Cardiovasc Surg 2003; 51(1):17-21. [CrossRef][PubMed]

26. Pattakos G, Koch CG, Brizzio ME, Batizy LH, Sabik JF 3rd, Blackstone EH, et al. Outcome of patients who refuse transfusion after cardiac surgery: a natural experiment with severe blood conservation. Arch Intern Med 2012; 172(15): 1154-60. [CrossRef] [PubMed]

27. Loor G, Li L, Sabik JF, 3rd, Rajeswaran J, Blackstone EH, Koch CG. Nadir hematocrit during cardiopulmonary bypass: end-organ dysfunction and mortality. J Thorac Cardiovasc Surg 2012; 144(3): 654-662. [CrossRef]

28. Paone G, Herbert MA, Theurer PF, Bell GF, Williams $\mathrm{JK}$, Shannon $\mathrm{FL}$, et al. Red blood cells and mortality after coronary artery bypass graft surgery: an analysis 
of 672 operative deaths. Ann Thorac Surg 2015; 99(5): 1583-9. [CrossRef][PubMed]

29. Shaw RE, Johnson CK, Ferrari G, Zapolanski A, Brizzio $M$, Rioux $N$, et al. Balancing the benefits and risks of blood transfusions in patients undergoing cardiac surgery: a propensity-matched analysis. Interact Cardiovasc Thorac Surg 2013; 17(1):96-102. [CrossRef][PubMed]

30. Kuduvalli M, Oo AY, Newall N, Grayson AD, Jackson M, Desmond $\mathrm{MJ}$, et al. Effect of peri-operative red blood cell transfusion on 30-day and 1-year mortality following coronary artery bypass surgery. Eur ] Cardiothorac Surg 2005; 27(4): 592-8. [CrossRef][PubMed]

31. Engoren MC, Habib RH, Zacharias A, Schwann TA, Riordan CJ, Durham SJ. Effect of blood transfusion on long-term survival after cardiac operation. Ann Thorac Surg 2002; 74(4): 1180-6. [CrossRef][PubMed]

32. Möhnle P, Snyder-Ramos SA, Miao Y, Kulier A, Böttiger BW, Levin J, et al. Postoperative red blood cell transfusion and morbid outcome in uncomplicated cardiac surgery patients. Intensive Care Med 2011; 37 (1): 97-109. [CrossRef][PubMed]

33. Loor G, Rajeswaran J, Li L, Sabik JF 3rd, Blackstone $\mathrm{EH}, \mathrm{McCrae} \mathrm{KR}$, et al. The least of 3 evils: exposure to red blood cell transfusion, anemia, or both? J Thorac Cardiovasc Surg 2013; 146(6): 1480-1487. [CrossRef][PubMed]

34. Schwann TA, Habib JR, Khalifeh JM, Nauffal V, Bonnell M, Clancy C, et al. Effects of Blood Transfusion on Cause-Specific Late Mortality After Coronary Artery Bypass Grafting-Less Is More. Ann Thorac Surg 2016; 102(2): 465-73. [CrossRef][PubMed]

35. Du Pont-Thibodeau G, Harrington K, Lacroix J. Anemia and red blood cell transfusion in critically ill cardiac patients. Ann Intensive Care 2014; 4: 16. [CrossRef][PubMed]

36. Azarfarin R, Ashouri N, Totonchi Z, Bakhshandeh $H_{\text {, }}$ Yaghoubi A. Factors influencing prolonged ICU stay after open heart surgery. Res Cardiovasc Med 2014; 3(4): e20159. [PubMed]

37. Mehta RH, Grab JD, O'Brien SM, Glower DD, Haan CK, Gammie JS, et al. Clinical characteristics and inhospital outcomes of patients with cardiogenic shock undergoing coronary artery bypass surgery: insights from the Society of Thoracic Surgeons National CardiacDatabase. Circulation 2008; 117(7): 876-85. [CrossRef][PubMed]

38. Jubelirer SJ, Mousa L, Reddy U, Welch CA. Coronary artery bypass grafting (CABG) in patients with immune thrombocytopenia (ITP): a community hospital experience and review of the literature. W V Med J 2011; 107(6): 10-4. [PubMed]

39. Engoren M, Arslanian-Engoren C. Long-term survival in the intensive care unit after erythrocyte blood transfusion. Am J Crit Care 2009; 18(2): 124-31. [CrossRef][PubMed]

40. Vamvakas EC, Carven JH. RBC transfusion and postoperative length of stay in the hospital or the intensive care unit among patients undergoing coronary artery bypass graft surgery: the effects of confounding factors. Transfusion 2000; 40(38): 832-9. [CrossRef][PubMed]

41. Koster A, Zittermann A, Borgermann J, Knabbe C, Diekmann J, Schirmer U, et al. Transfusion of 1 and 2 units of red blood cells does not increase mortality and organ failure in patients undergoing isolated coronary artery bypass grafting. Eur J Cardiothorac Surg 2016; 49(3): 931-6. [CrossRef][PubMed]

42. Haanschoten $M C$, van Straten $A H$, Verstappen $F$, van de Kerkhof D, van Zundert AA, Soliman Hamad MA. Reducing the immediate availability of red blood cells in cardiac surgery, a single-centre experience. Neth Heart J 2015; 23(1): 28-32. [CrossRef][PubMed]

43. Ferraris VA. Blood transfusion in cardiac surgery: who should get transfused? Lancet Haematol 2015; 2(12). [CrossRef][PubMed] 


\title{
POREMEĆAJI KOAGULACIONOG STATUSA I HEMOSTAZE KAO PROGNOSTIČKI PARAMETRI NEPOSREDNIH I RANIH REZULTATA NAKON HIRURŠKE REVASKULARIZACIJE MIOKARDA
}

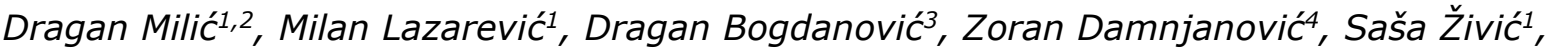 \\ Dejan Perić1 ${ }^{1}$ Aleksandar Kamenov ${ }^{1}$, Vladimir Stojiljković ${ }^{1}$, Mlađan Golubovićs
}

\author{
${ }^{1}$ Klinika za kardiohirurgiju, Klinički centar Niš, Niš, Srbija \\ ${ }^{2}$ Univerzitet u Nišu, Medicinski fakultet, Niš, Srbija \\ ${ }^{3}$ Institut za javno zdravlje Niš, Niš, Srbija \\ ${ }^{4}$ Klinika za vaskularnu hirurgiju, Klinički centar Niš, Niš, Srbija \\ ${ }^{5}$ Klinika za anesteziologiju, Klinički centar Niš, Nišs, Srbija
}

Kontakt: Dragan Milić

Bulevar dr Zorana Đinđića 81, 18000 Niš, Srbija

E-mail: drdraganmilic@gmail.com

Hirurška revaskularizacija miokarda predstavlja jednu od najčešće izvođenih hirurških procedura u svetu. Vremenom, razvojem tehnologije i savremenih dijagnostičkih postupaka kao i unapređenjem hirurške tehnike, stopa smrtnosti kod elektivnih nekomplikovanih slučajeva pala je na ispod $2 \%$. Ipak, i pored izuzetnog razvoja hirurške metode, stopa postoperativnih komplikacija koje mogu ugroziti bolesnike kreće se i preko $10 \%$. Cilj ovog istraživanja bio je da se definiše grupa bolesnika sa povećanim rizikom od postoperativnih komplikacija u zavisnosti od poremećaja koagulacionog statusa i hemostaze.

Sprovedeno je prospektivno, nerandomizovano istraživanje koje je obuhvatilo 28 bolesnika koji su podvrgnuti hirurškoj revaskularizaciji miokarda u Klinici za kardiohirurgiju KC Niš od januara do aprila meseca 2017. godine. Preoperativno, kao i tri sata, 24 sata, 48 sati, tri dana i pet dana postoperativno, određivani su sledeći parametri: krvna slika, parametri inflamacije (C reaktivni protein, presepsin); koagulacioni status (protrombinsko vreme, International Normalized Ratio, aktivisano parcijalno tromboplastinsko vreme, fibrinogen, anti-trombin III, D dimer).

Jedini preoperativni nezavisni prognostički parametar povećane postoperativne drenaže bio je INR. ACT je bio nezavisni postoperativni prognostički parametar povećane postoperativne drenaže verovatno zbog odloženog ili protrahovanog dejstva heparina. Parametri inflamacije nisu pokazali povezanost sa nastankom postoperativnih komplikacija. $U$ odnosu na bolesnike bez krvarenja, kod onih sa krvarenjem evidentirane su značajno više vrednosti uree i razlike vrednosti APTT preoperativno i na kraju perioda praćenja. Multivarijantna logistička regresiona analiza je kao jedini faktor značajno povezan sa rizikom za nastanak krvarenja potvrdila razliku vrednosti APTT preoperativno i na kraju perioda praćenja. Multi-varijantna linearna regresiona analiza je kao jedini faktor značajno povezan sa promenom vrednosti ukupno date alogene transfuzije potvrdila vrednost uree. Povećanje nivoa uree povezano je sa porastom vrednosti ukupno date alogene transfuzije. Korelaciona analiza je pokazala da je povećan broj dana boravka u intenzivnoj nezi bio značajno povezan sa ženskim polom, povećanjem broja graftova i povišenim vrednostima trajanja EKK, vremena klemovanja, vrednosti HCT, PT, INR preoperativno i na kraju perioda praćenja.

Hirurška revaskularizacija miokarda je bezbedna i sigurna metoda sa minimalnom stopom morbiditeta. Primenom savremenih metoda za preoperativni monitoring hemostaze može se značajno smanjiti rizik postoperativnog krvarenja i smanjiti potreba za transfuzijom crvenih krvnih zrnaca i drugih derivata krvi.

\section{Acta Medica Medianae 2019;58(1):64-81.}

Ključne reči: kardiohirurgija, koagulacioni status, parametri inflamacije, faktori rizika 